\title{
Sonic Intelligence as a Virtual Therapeutic Environment
}

\author{
IOANNIS TARNANAS, M.Sc., and DIMITRIOS ADAM, M.Sc.
}

\begin{abstract}
This paper reports on the results of a research project, on comparing one virtual collaborative environment with a first-person visual immersion (first-perspective interaction) and a second one where the user interacts through a sound-kinetic virtual representation of himself (avatar), as a stress-coping environment in real-life situations. Recent developments in coping research are proposing a shift from a trait-oriented approach of coping to a more situation-specific treatment. We defined as real-life situation a target-oriented situation that demands a complex coping skills inventory of high self-efficacy and internal or external "locus of control" strategies. The participants were 90 normal adults with healthy or impaired coping skills, 25-40 years of age, randomly spread across two groups. There was the same number of participants across groups and gender balance within groups. All two groups went through two phases. In Phase I, Solo, one participant was assessed using a three-stage assessment inspired by the transactional stress theory of Lazarus ${ }^{1}$ and the stress inoculation theory of Meichenbaum. ${ }^{2}$ In Phase I, each participant was given a coping skills measurement within the time course of various hypothetical stressful encounters performed in two different conditions and a control group. In Condition $A$, the participant was given a virtual stress assessment scenario relative to a first-person perspective (VRFP). In Condition B, the participant was given a virtual stress assessment scenario relative to a behaviorally realistic motion controlled avatar with sonic feedback (VRSA). In Condition C, the No Treatment Condition (NTC), the participant received just an interview. In Phase II, all three groups were mixed and exercised the same tasks but with two participants in pairs. The results showed that the VRSA group performed notably better in terms of cognitive appraisals, emotions and attributions than the other two groups in Phase I (VRSA, 92\%; VRFP, 85\%; NTC, 34\%). In Phase II, the difference again favored the VRSA group against the other two. These results indicate that a virtual collaborative environment seems to be a consistent coping environment, tapping two classes of stress: (a) aversive or ambiguous situations, and (b) loss or failure situations in relation to the stress inoculation theory. ${ }^{3}$ In terms of coping behaviors, a distinction is made between self-directed and environment-directed strategies. A great advantage of the virtual collaborative environment with the behaviorally enhanced sound-kinetic avatar is the consideration of team coping intentions in different stages. Even if the aim is to tap transactional processes in real-life situations, it might be better to conduct research using a sound-kinetic avatar based collaborative environment than a virtual first-person perspective scenario alone. The VE consisted of two dual-processor PC systems, a video splitter, a digital camera and two stereoscopic CRT displays. The system was programmed in $\mathrm{C}^{++}$and VRScape Immersive Cluster from VRCO, which created an artificial environment that encodes the user's motion from a video camera, targeted at the face of the users and physiological sensors attached to the body.
\end{abstract}

Department of Psychology and Informatics, Aristotle University of Thessalonica, Thessalonica, Greece. 


\section{INTRODUCTION}

$\mathbf{T}$ HE USE OF VIRTUAL REALITY (VR) to develop new diagnostic tools for psychology and neuropsychology has been proposed and discussed in many research groups ${ }^{3-6}$ since the early 1990s. Basically, VR is suggested as a means of assessing aspects of behavior that are normally inaccessible during traditional formal psychometric testing. In fact, VR-based tests are thought to be more representative of everyday life situations than paperand-pencil (p\&p) tests, but potentially as precise and reliable as the latter. A primary necessity is, therefore, the demonstration that VR adds value to already existing neuropsychometric tools. This would imply, for example, that new tools are better in terms of sensitivity to specific cognitive deficits than older ones. To some extent, therefore, new products need to be compared with older but reliable and validated tools. A straightforward approach would be to test the same subjects on both traditional and VR-based tools and compare the results. This, however, is complicated by the fact that VR-and especially immersive VR-changes substantially the cognitive requests of a given test. For example, the amplification of spatial, motor and time-related aspects of a task that unavoidably takes place in a virtual reality may change in an unpredictable way the response values and their distribution, even though the strategic aspects are kept constant. It has been therefore anticipated that VR-based tests will evolve as a new class of diagnostic tools ${ }^{7}$ with psychometric characteristics substantially different from their $p \& p$ analogs.

Our two groups in this experiment are involved in a research project dealing with the development of new VR-based tools and methodologies to assess and retrain acquired coping skill strategies. We are focusing particularly on coping skills that arise as a consequence of stress and psychosocial—workrelated-disorders. This paper summarizes the work that has been carried out so far to assess situation specific psychosocial coping skills. Using a Distributed Cognition theoretical framework, we tried to map interpersonal interactions and assess whether the coping behaviors in a collaborative virtual environment are self-directed or environment-directed strategies. A behaviorally realistic sound-kinetic avatar is a virtual representation of the user's body motor coordination and balance in an artificial agent, which gives a real-time sonic feedback to the actual movements of the user, using a video camera and on-the-body sensors. The sonic feedback is based on a custom musical protocol and system that is described elsewhere. ${ }^{8}$

\section{MATERIALS AND METHODS}

\section{The Virtual Environmental design and implementation}

The VR work-related stress scenario was developed using a custom virtual reality system at the Aristotle University of Thessalonica, Department of Psychology. The system was a Pentium III-based immersive VR system (700 Mhz, $128 \mathrm{Mb}$ RAM, graphic engine of Matrox G400 Dual Head, $32 \mathrm{Mb}$ WRam), including two CRT display subsystems (Kaiser Proview XL35/50) and some EEG sensors. The visual immersion is realized through the rear projection and active stereo. The trainee wears lightweight (compared to HMD) shutter glasses and faces the screen of the $\sim 3.0 \times 2.8 \mathrm{~m}$ size. Due to the rear projection, he is able to approach the screen very closely without any shadow being cast on the screen. Compared to HMD this solution is less cumbersome, we avoid problems of very limited field of view, cyber sickness (related to the lack of peripheral vision) and claustrophobic effects in case of some more sensitive trainees.

Navigation paradigm is based on a single magnetic tracker attached to the trainee's head. Ascensions PC Bird is used for this purpose. In order to "walk around" the virtual environment, the trainee needs to step into the navigation ring, which in effect triggers camera motion in the desired direction. The trainee can still move inside the central area of the ring without causing any camera motion. In order to "look around," the trainee needs to look at the margins of the projection screen. This analogously triggers horizontal and vertical camera rotation. The paradigm is lightweight and intuitive to understand. Moreover following the requirement of the modularity and scalability the magnetic tracker can be replaced by a wireless hand-held mouse or a normal mouse in case of scale down.

The virtual coping skills scenario is a virtual collaborative environment developed using the VRScape Immersive Cluster from VRCO. The main attraction is the interior of a work office within a large virtual organization. The possible variations include different work roles, a highly motivated director and some special hostile environment situations that are extremely stressful. The therapist constantly monitors the session using a Multidimensional Health Profile-Psychosocial (MHP-P) 


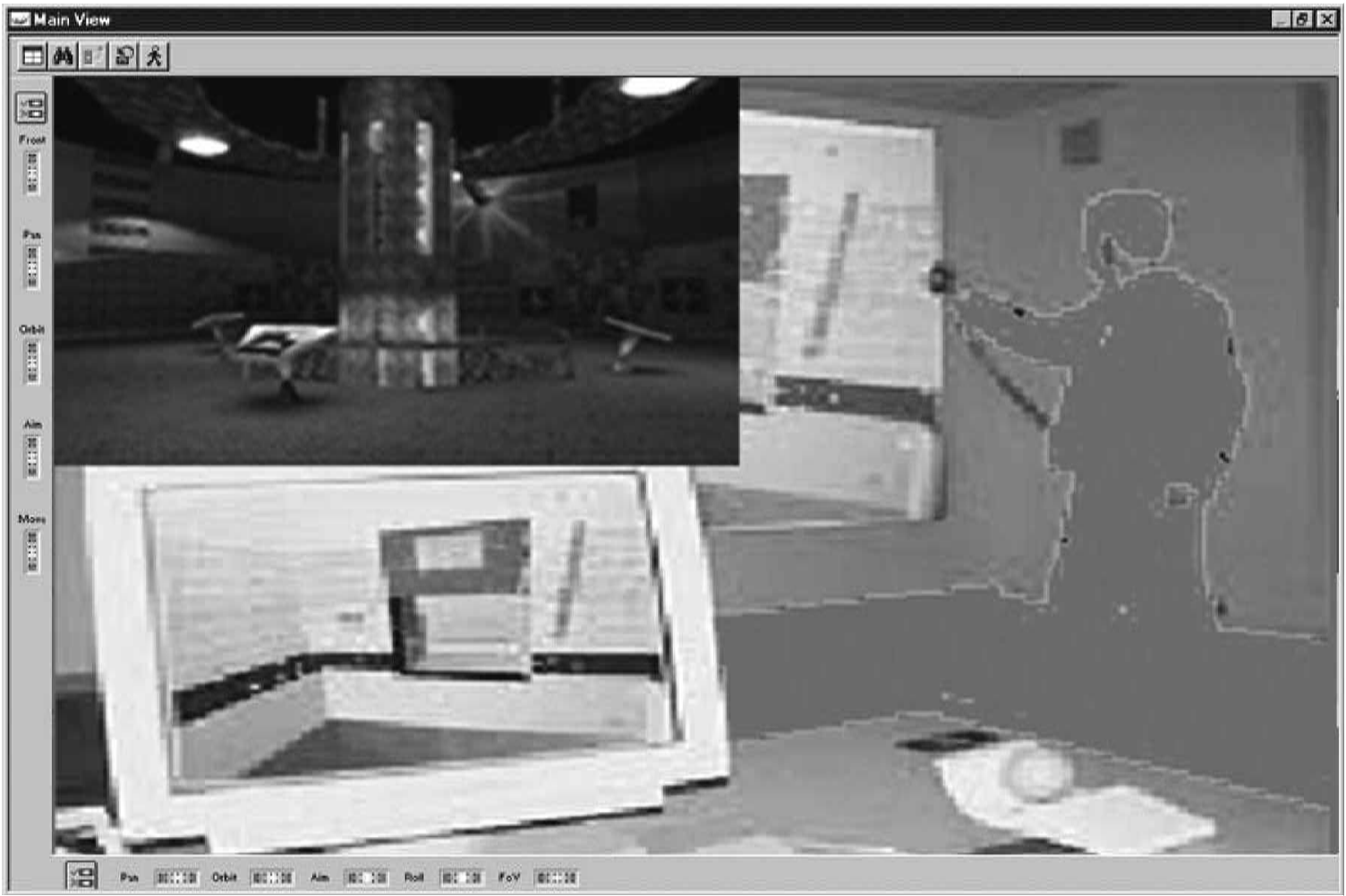

FIG. 1. Image showing the sound-kinetic representation of one's self, as seen inside the Virtual Collaborative Environment. The participant is experiencing a high demanding virtual environment (seen at the left top corner) and an artistic representation of himself created in real time that targets his Situational Awareness. More information can be found at http:/ / users.auth.gr/ ioannist.

Score Report, ${ }^{9}$ modeling the emotional and behavioral responses of the subjects. In particular, the therapist can define the length of the virtual experience, its end and the cues that the scenario is going to propose according to the phase of the session.

\section{The methodology}

We have then developed a VR analog based on the Stress and Coping Process Questionnaire (SCPQ), and of the most commonly used "coping skills assessment" tests. ${ }^{10}$ The writer has described the project in detail in previous papers, and its application to a clinical case has been recently discussed. ${ }^{11}$ Here, we are presenting results concerning psychometric aspects of the performance of healthy coping strategies and coping-impaired individuals (work-related stress disorder), and the relationships between the two test versions.

Methods. The virtual environment features only two very simple architectural modules: rooms and connecting corridors that resemble a real-life work environment. While in a room, subjects are asked to move about by opening one of several doors that contained some stress scenes from the three categories of the transactional stress and coping process questionnaire (SCPQ). The subjects' task is to proceed by trial and error until they find the doors that lead outside of the work offices building. Each subject is expected to develop a strategy to avoid the frustration of frequent failure. Subjects face a commercial good-resolution stereoscopic CRT display and stand inside a virtual ring that served to navigate the virtual environment and interact with the doors. They were given a maximum of $45 \mathrm{~min}$ to complete their journey through 32 virtual rooms. The software kept a record of a number of events and their time of occurrence.

Subjects. Forty-five healthy subjects and 45 patients with work-related stress disorders volunteered for this study. In the patients' group, there were 25 subjects with aggressive behavior and 20 with neurological sequelae of cerebrovascular accidents. Controls and patients did not differ for mean age and years of formal education. Subjects were tested in a counterbalanced order on the VRSA 
and on the VRFP. An external experimenter (A.M.) who was unaware of the subjects' clinical diagnosis performed the scoring. ${ }^{12}$ Errors were not further classified because of the difficulty of equating perseverative events that occurred in a VR setting. The square root of the ratio between all correct responses and errors was taken to compute summary indexes of performance on the two tests.

Results. Controls had higher summary index values than patients on both the VRFP test and its VRSA analog. Within each group, however, there was a difference between those who were given first the VRSA analog and those who got the VRFP test first (Table 1). On average, patients attained less categories, gave more responses of the "correct" type and made more errors than healthy subjects on the VRFP; on the VRSA analog, they also attained less categories and made more errors. The way subjects learned the strategy was, however, markedly different between the two tests. On the VRFP, the average number of errors to attain successive stress and coping process categories increased almost linearly up to the fourth or the fifth set for the controls and patients, respectively. On the VRSA test, the average number of errors decreased steeply, going from the first to the second and third categories. Within each test, patients differed from controls on the number of trials to attain a given category, not on the overall pattern of response. Summary index values were used for this analysis. The strength of the linear correlation computed over the whole sample $(n=64)$ was modest (Pearson's $r=0.44$ ) but statistically significant $(p=0.001)$; patients' values were less scattered around the regression line than controls' values, but the two groups had identical slopes. Finally, the number of attained criteria, and the total correct and error responses for each test were used as input for discriminant analyses aimed at assessing the potential to distinguish coping skills intact from work-related stress-coping skills-impaired subjects. Two discriminant functions were extracted from each analysis. Overall hit rates for the VRFP and the VRSA tests were $60.1 \%$ and $71.4 \%$, respectively. In particular, more patients were correctly classified by the VRSA than by the p\&p test $(92 \%$ vs. $85 \%$ ). These results should be interpreted as a very preliminary and empirical application, since we have made no attempts to optimize the equations or to confirm their performance on new cases.

\section{Study II}

The previous study could not separate the influence of a group versus individual participation in a VR-based task, nor could control for possible transfer of learning from one SCPQ task to the other. Particularly, the first issue is a relevant one since the interaction with a virtual environment is never intuitive or fully natural-except maybe when stress crisis situations are simulated as in the first experiment. For a number of other situations, however, the user must learn how to operate an uncommon input device such as the navigation ring. This may have a limited yet considerable cost in terms of cognitive demands and resources available to carry out the primary task. This concern may be even more justified when TBI subjects are tested. Our group at Aristotle University of Thessaloniki has carried out a second experiment to show the differential effect on group-team or individual participant.

Method. The same 120 subjects as in the first study participated again (mean age, 36; SD 11.5 years). The study utilized a virtual environment comprised of four rooms in a large virtual organization.

Table 1. Demographic Data And Distribution of Summary IndeXesa According to Diagnostic Group and Order of TeSt AdMINISTRATION

\begin{tabular}{|c|c|c|c|c|c|c|}
\hline & \multicolumn{2}{|c|}{ Controls $(n=60)$} & \multicolumn{2}{|c|}{ Patients $(n=60)$} & \multicolumn{2}{|c|}{$2 \times A N O V A$} \\
\hline & Mean & $S D$ & Mean & $S D$ & $F$ & $p$ \\
\hline Age (years) & 32.6 & 8.4 & 36.4 & 11.5 & & \\
\hline Education (years) & 12 & 2.8 & 12.1 & 3.2 & & \\
\hline \multicolumn{7}{|l|}{ Alone } \\
\hline VRFP first & 3.23 & 1.10 & 2.72 & 1.32 & & \\
\hline VRSA first & 3.48 & 1.42 & 2.96 & 1.30 & 4.2 & 0.04 \\
\hline \multicolumn{7}{|l|}{ Team } \\
\hline VRFP first & 1.69 & 1.01 & 1.16 & 0.48 & & \\
\hline VRSA first & 1.89 & 0.75 & 1.36 & 0.82 & 5.4 & 0.02 \\
\hline
\end{tabular}

aSummary index: the square root of the ratio between total correct responses and total errors. 
Twenty-five decision-making tasks were situated in these rooms. Subjects were asked to explore the rooms in search of a specific task that, in fact, was not there. They could move inside the virtual organization at a CAVE-like setting (immersive VR setup). This VR setting was again a VRFP and a VRSA, but with a more collaborative design. The SCPQ test was adjusted for this study and a factor analysis was carried out in order to match the transactional stress theory of Lazarus in a collaborative design. Participants were randomly allocated to the group or individual condition and tested in "yoked" pairs without actually knowing it. Immediately after completion of the route through the virtual house, both members of a pair were asked to complete the SCPQ analog of the collaborative coping skills VR setting.

Results. Contrary to expectations, healthy subjects who were exploring the VE in groups did not score worse than their "individual" VR test (mean probability, 0.51 , SD 0.12 , vs. 0.51, SD 0.15). Group participants were better than individual members of yoked pairs in all the task of the VRFP categories analogous to this virtual setting (mean probability), 0.58 , SD 0.17 , vs. 0.45 , SD 0.15 ). A $2 \times 2$ ANOVA for repeated measures confirmed a significant effect of healthy coping skills versus work-related stress ( $\mathrm{df}$ 1.24; $F=80.4 ; p<0.001)$. The interaction between role and type of coping task fell short of significance $(F=3.72 ; p=0.06)$.

Comment. This study confirms that the group VR test that we have developed has psychometric properties comparable to those of the individual scenarios, from which they are derived. Thus, it can distinguish patients from healthy subjects at least as well as the latter, though there are hints that it can do better. The fact that there is no transfer of implicit knowledge from one test to the other supports the hypothesis that VR-based tests have specific characteristics. Accordingly, we have found only weak correlations between summary scores obtained by using the two variants. Relevant to this issue is also the demonstration that the learning process that underlies the acquisition of a correct strategy takes a different course in the two tests. We suggest that this finding depends on the more complex (and complete) cognitive demands of the VR setting at the beginning of the test when perceptuomotor, visuospatial (orientation), memory and conceptual aspects of the task need to be fully integrated into an efficient routine. We have previously provided ancillary evidence that a successful performance on the VR test depends on such an integration. ${ }^{13}$ On the VRFP, instead, the initial conceptualization is attained relatively easily and little additional difficulty is met until an anger management event is met, when a rather counterintuitive "restart" at the point of view has to be made. Of course, these conclusions are pertinent to the study groups we have examined and cannotat the present time-be generalized. Finally, this study provides further evidence that an immersive VR system can be safely used with groups of individuals with a variety of mildly to moderately severe neurological and cognitive impairments.

\section{DISCUSSION}

VR technology could potentially improve the reliability of neuropsychological assessment by allowing for more consistent presentation and manipulation of complex test stimuli along with more precise measurement of participant responses. The reliability and validity of measurement of the component cognitive domains of attention could potentially be enhanced by the capacity of VR technology to present both test and distraction stimuli along with better quantification of discrete responding. In this manner, VR could offer the potential for cognitive assessment and rehabilitation within simulated "real-world" functional testing and training environments with an aim towards improving ecological validity. A more precise form of measuring attention performance within a standard theoretical framework, like distributed cognition, using VEs modeled after real-life settings should, in theory, ${ }^{14}$ provide better predictions of performance in the real world. Distributed cognition is a theoretical framework that differs from mainstream cognitive science by not privileging the individual human actor as the unit of analysis. Distributed cognition acknowledges that in a vast majority of cases cognitive work is not being done in isolation inside our heads but is distributed among people, between persons and artifacts, and across time. What makes a system cognitive is the presence of processes applied to representational states that result in cognitive work. Tracking the representational states using a behaviorally realistic sound-kinetic avatar (agent) can uncover the specific cognitive processes being employed. Our collaborative virtual environments, though designed to assess coping behaviors directed to work-related disorders, could potentially act as preventive optimism training (cognitive behavioral therapy) for improved health behaviors, service 
utilization, and depression. ${ }^{15}$ Following empirical testing of the parameters outlined above, our plan is to develop an inexpensive system that would be used in clinics, schools, and research settings. While we are currently using high-end equipment we hope that, by the time we have empirically developed a reliable and valid set of VR tasks and basic clinical trials are conducted to develop normative data, the technology will have advanced to the point where our scenario could be delivered on less expensive and readily available equipment.

\section{REFERENCES}

1. Lazarus, R.S. (1991). Cognition and motivation in emotion. American Psychologist 46:352-367.

2. Bowers, D., \& Meichenbaum, D. (1994). The unconscious reconsidered. New York: Wiley.

3. Rose, F.D., Attree, E.A., \& Johnson, D.A. (1996). Virtual reality: an assistive technology in neurological rehabilitation. Current Opinion in Neurology 9: 461-467.

4. Rizzo, A.A., Buckwalter, J.G., \& Neumann, U. (1997). Virtual reality and cognitive rehabilitation: a brief review of the future. Journal of Head Trauma Rehabilitation 12:1-15.

5. Johnson, D.A., Rushton, S., \& Shaw, J. (1996). Virtual reality enriched environments, physical exercise and neuropsychological rehabilitation. Presented at the 1st European Conference on Disability, Virtual Reality and Associated Technologies, Maidenhead.

6. Pugnetti, L., Mendozzi, L., Motta, A., et al. (1995). Evaluation and retraining of adults' cognitive impairments: which role for virtual reality technology? Computers in Biology and Medicine 25:213-228.

7. Pugnetti, L., Mendozzi, L., Motta, A., et al. (1995). Immersive virtual reality to assist retraining of acquired cognitive deficits: first results with a dedicated system. In: Satava, R.M., Morgan, K., Sieburg, S.H., et al. (eds.), Interactive technology and the new paradigm for healthcare. Amsterdam: IOS Press, pp. 289-297.

8. Tarnanas, I.A., \& Kikis, V. (2002). Sound-kinetic feedback for virtual therapeutic environments. Presented at the ICDVRAT 2002 conference.

9. Ruehlman, L.S., Lanyon, R.I., and Karoly, P. (1998). Multidimensional Health Profile-Psychosocial (MHPP) Score Report. Lutz, FL: Psychological Assessment Resources, Inc.

10. Perrez, M., \& Reicherts, M. (1992). Stress, coping, and health. Seattle, WA: Hogrefe \& Huber.

11. Tarnanas, I., \& Manos, G. (2001). Using virtual reality to teach special populations how to cope in crisis: the case of a virtual earthquake. Presented at the Medicine Meets Virtual Reality Conference.

12. Mendozzi, L., Motta, A., Barbieri, E., et al. (1997). The application of virtual reality to document coping deficits after a stroke: report of a case. CyberPsychology $\mathcal{E}$ Behavior.

13. Pugnetti, L., Mendozzi, L., Barbieri, E., et al. (1996). Nervous system correlates of virtual reality experience. Presented at the 1st European Conference on Disability, Virtual Reality and Associated Technologies, Maidenhead.

14. Decety, J., Perani, D., Jeannerod, M., et al. (1994). Mapping motor representations with positron emission tomography. Nature 371:600-602.

15. Buchanan, G.M., Rubenstein, C.A., \& Seligman, M.E.P. (1999). Physical health following a cognitivebehavioral intervention. Prevention $\mathcal{E}$ Treatment. Online: http://journals.apa.org/prevention/volume2/ pre0020010a.html.

Address reprint requests to: Ioannis Tarnanas, M.Sc.

Department of Psychology and Informatics Aristotle University of Thessalonica Thessalonica, Greece

E-mail: ioannist@psy.auth.gr 